\title{
Evolución histórica de las vacunas contra el Virus Papiloma Humano
}

Esta larga y exitosa historia cada día avanza un poco más. En más de 20 años, ella incluye una serie de eventos que comienzan con el establecimiento de una relación de gran fuerza entre una infección viral (la infección persistente del tracto genital inferior por el Virus Papiloma Humano o VPH) y una serie de patologías de alto impacto social y con gran repercusión en morbilidad y mortalidad, en particular para la mujer.

En el año 2003 durante el Congreso Mundial FIGO realizado en Chile, el Dr. Xavier Bosch presentó una conferencia llamada "Cáncer cervicouterino: de una enfermedad oncológica a una enfermedad infecciosa". Muchos de los allí presentes observamos con incredulidad la evidencia presentada, reinó en muchos el escepticismo pese a la fuerza de los datos epidemiológicos. La historia continuó y dio paso a la necesidad de estudiar y aprender más sobre la asociación epidemiológica más potente hasta entonces conocida, entre un agente causal (VPH) y un evento final devastador (cáncer de cuello uterino). Así del escepticismo se pasó al reconocimiento, hecho que queda reflejado en el otorgamiento del premio Nobel de Medicina en 2008 al Profesor Dr. Harald Zur Hausen, médico y científico alemán, quien condujera los estudios que llevaron a descubrir y demostrar esta asociación. Hoy tenemos un conocimiento más acabado de la historia natural del cáncer de cuello, incluyendo un entendimiento mayor de los mecanismos que controlan la infección del epitelio cervical por VPH y los eventos moleculares que promueven la carcinogénesis. Ello ha permitido el desarrollo de nuevas técnicas moleculares de tamizaje, probablemente más sensibles y complementarias a la citología cervical, que sin lugar a dudas permitirán mejorar las estrategias de prevención secundaria del cáncer de cuello uterino. Así también, el desarrollo y validación de vacunas efectivas en su prevención primaria.

Hoy sabemos con certeza que la infección por el VPH constituye la infección de transmisión sexual más frecuente, tanto en mujeres como en hombres, y que son fuente de variada patología tanto benigna como maligna para ambos sexos. Si bien la mayoría de las infecciones son asintomáticas y se resuelven espontáneamente, es la infección persistente por los genotipos oncogénicos (especialmente los 16 y 18) la que puede evolucionar a enfermedad preinvasora la cual, si no es diagnosticada y tratada adecuadamente, determina la aparición de cáncer.

Dentro de las enfermedad asociadas a la infección por VPH se incluyen las siguientes: verrugas genitales, lesiones pre-malignas de vulva, vagina, cuello del útero y ano, cáncer de vulva, vagina y cuello del útero, cáncer de ano, cáncer de pene, cáncer de boca y faringe, papilomatosis laríngea, y papilomatosis recurrente de las vías respiratorias (laringe, tráquea y bronquios).

Desde la perspectiva ginecológica, la infección por VPH es una condición de alta prevalencia, afectando aproximadamente entre 1 a 2 de cada 10 mujeres en nuestro país. Ello determina un número importante de casos anuales de condilomatosis vulvar y perianal (aproximadamente el $2 \%$ de la población sexualmente activa), los cuales afectan más comúnmente a mujeres jóvenes. Junto a ello, y pese a la implementación del programa de tamizaje con papanicolau, alrededor de 1200 mujeres son diagnosticadas con un cáncer de cuello uterino y poco menos de 600 de ellas mueren por esta causa, determinando una mortalidad de 6,7 por 100.000 mujeres (Registro Nacional de Cáncer, 2012). A nivel mundial, los números resultan aún más dramáticos en términos de cáncer. Anualmente se diagnostican 500.000 casos nuevos de cáncer de cuello uterino y 270.000 mujeres fallecen por esta causa, el $85 \%$ de ellas en los países más pobres.

La asociación entre VPH y cáncer de cuello uterino llevó a diversos investigadores a avanzar en el diseño y el desarrollo de vacunas destinadas a evitar el contagio por el virus y así evitar la infección persistente del epitelio cervical, evento crucial de la carcinogénesis cervical. Dos investigadores, 
Ian Frazer y Jian Zhou, lograron ensamblar partículas proteicas similares al virus (Viral Like Particles o VLP) con capacidad antigénica, a partir de la cubierta o envoltura que protege la doble hebra de ADN, permitiendo el desarrollo de las vacunas que actualmente conocemos. A partir del año 2006 y luego de diferentes estudios clínicos que validaron su eficacia en la prevención en el desarrollo de lesiones premalignas y de condilomas, 2 vacunas contra el VPH están disponibles para su aplicación.

Las vacunas actualmente disponibles son dos, la bivalente que protege contra los genotipos oncogénicos 16 y 18 (Cervarix®) y la tetravalente que adicionalmente a los genotipos 16 y 18 inmuniza contra los genotipos 6 y 11, responsables del $90 \%$ de la verrugas genitales (Gardasil囚). Ambas vacunas son profilácticas y generan una respuesta inmune potente, muy superior a la que se obtendría con la infección natural. Se estima que los niveles de anticuerpos alcanzados son 10 veces mayores a los inducidos por dicha infección. Todas las adolescentes y mujeres jóvenes que nunca han estado expuestas al virus previamente, desarrollan una respuesta de anticuerpos al cabo de tres dosis y además inducen una respuesta de memoria inmunológica mediada por células $B$. Dicha respuesta inmunológica es mayor a menor edad. Ello explica que la vacuna se coloque en niñas o adolescentes más que en mujeres adultas.

Desde su autorización por la Food and Drug Administration (FDA) y otros organismos sanitarios internacionales, diversos grupos de la comunidad han mostrado oposición a su implementación. Dentro de los argumentos que se esgrimen están el alto costo que implica su incorporación a los programas de vacunación, que lo haría una medida no costo efectiva, los riesgos potenciales de mortalidad vinculada a su administración y la falta de evidencia concreta que demuestre su impacto real, a largo plazo, en reducir la mortalidad por cáncer de cuello uterino.

Sin embargo, para cada uno de estos argumentos la evidencia ha ido demostrando paulatinamente que se trata de temores infundados. De hecho los estudios de costo efectividad ya la avalan como medida sanitaria en países con diferentes ingresos per cápita, con o sin programas de tamizaje efectivos y con distinta prevalencia de cáncer de cuello uterino. Respecto de su seguridad, tanto las VLP como los inductores de inmunogenicidad que le acompañan, han mostrado un adecuado perfil de bioseguridad. Es así que tanto la Federación Internacional de Obstetricia y Ginecología (FIGO) como la Organización Mundial de la Salud (OMS) han dado fe de ello, tal como se comprueba en la publicación de marzo de este año del Comité Asesor Global de Seguridad de Vacunas de OMS donde se señala que "ambas vacunas contra el VPH continúan teniendo un excelente perfil de seguridad".
Por último, el seguimiento disponible a la fecha, en particular lo reportado por los grupos australianos, donde se registran las primeras cohortes de mujeres vacunadas, hace ya 8 años, parecieran comenzar a confirmar la eficacia de las vacunas. Es aceptable y entendible que exista escepticismo sobre su real efectividad mientras no se demuestre la reducción de cáncer de cuello uterino en la cohorte vacunada. Demostrar dicho efecto en incidencia y mortalidad demorara varias décadas, pero se puede establecer una línea de tiempo con beneficios a corto plazo (reducción de verrugas y disminución de la tasas de infección por el virus) y de mediano plazo como la reducción de lesiones cervicales, de citologías positivas y de procedimientos relacionados.

En este sentido la experiencia australiana con vacunación de niñas y mujeres jóvenes entre los años 2007 y 2009 ha demostrado la casi desaparición de verrugas genitales a los 4 años de seguimiento en mujeres menores de 21 años, así como también en varones heterosexuales menores de 21 (efecto rebaño) y a la vez una tasa estable entre hombres que tienen sexo con hombres (todos los cuales hasta entonces no habían sido vacunados). En relación a lesiones pre invasoras de cuello, ambas vacunas han demostrado eficacias cercanas a $100 \%$ en la disminución de NIE II, NIE III y adenocarcinoma in situ (AIS) asociadas a los genotipos vacunales, eficacia que se mantiene en el largo plazo como lo muestra el estudio de los países nórdicos donde se obtiene $100 \%$ de protección contra NIE III y AIS dentro de la población con apego al protocolo.

Una vez confirmada la eficacia de dichas vacunas, su seguridad y muy baja incidencia de efectos adversos graves, diversos países han comenzado a implementar programas de vacunación. Desde la primera cohorte de mujeres vacunadas en Australia, la implementación de dichos programas de vacunación se ha ido incrementado paulatinamente con el paso de los años y ya son 67 países los que la incluyen en su calendario de vacunación gubernamental, ya sea como programas organizados o como vacunación oportunista. En la actualidad se estima que se vacunan anualmente cerca de 115 millones de mujeres. Sin embargo, sólo el $15 \%$ de ellas corresponden a los países con más bajos recursos y con mayor mortalidad por este cáncer..... no deja de ser una gran incongruencia y una grave señal de inequidad a nivel mundial.

Sin embargo, no todo el conocimiento está completamente definido respecto a las vacunas. Aún existen controversias no esclarecidas totalmente. Por ejemplo, en lo relativo a protección cruzada, vale decir la protección inmunogénica contra genotipos diferentes a los que la vacuna posee; si bien ambas vacunas parecen diferir en este aspecto, siendo más amplia y de mayor magnitud para 
la vacuna bivalente, la significancia clínica y duración de esta respuesta no es clara. Así mismo el concepto de vacunación universal (tanto a varones como mujeres) parece no tener relevancia en lo concerniente a una mayor protección a la mujer y solo se indica como un propósito directo relacionada a disminuir la carga de enfermedad que afecta directamente a ellos. Así también parece ser más relevante donde existe alta movilidad o migración entre países de la población masculina.

Un aspecto relevante al momento de introducir la vacuna en los calendarios de vacunación y que se ha observado ampliamente en los diferentes países donde se ha intentado implementar el programa, es la dificultad de completar un esquema de tres dosis, lo que ha generado una necesidad de contar con esquemas más fáciles de aplicar y que generen mayor adhesividad en la población a vacunar. Coincidiendo con la observación de una respuesta inmunológica mas robusta en menores de 14 años, se ha generado un esquema de dos dosis basado en el principio de la equivalencia inmune, que implica que si la respuesta inmune en la población de 9 a 14 años con dos dosis no es inferior a la de la población de 15 a 25 años con tres dosis, la eficacia de un esquema puede ser atribuido al otro. En este aspecto la certeza solo consta en lo referente a una respuesta de inmunogenicidad en mujeres de 9 a 14 años inmunocompetentes y no tiene estudios de eficacia que la avalen, es por ello que en dicha población es imprescindible mantener la vigilancia epidemiológica a fin de evaluar la necesidad futura de una tercera dosis por ejemplo a los 5 años (esquemas extendidos).

La OMS ha entregado sus recomendaciones en relación a la vacuna VPH estableciendo la importancia de generar programas organizados de vacunas, idealmente en los colegios, acompañado de estrategias de educación a nivel de los medios de comunicación, esquemas con 2 dosis a 0 y 6 meses ó 0 y 12 meses a mujeres entre 9 y 13 años y que no se descuiden los programas actuales de tamizaje y de tratamiento de lesiones pre invasoras e invasoras. La vacuna a elegir debe depender de las realidades locales de cada país.

¿Cuál es la situación en nuestro país? Al respecto debemos señalar que ambas vacunas están licenciadas en Chile hace ya cerca de 7 años y su distribución había sido en forma oportunista y fundamentalmente limitada por el precio de ellas. Existiendo estudios de costo efectividad que demostraban que aun precio adecuado (US \$18 por dosis) su inserción dentro del programa nacional resultaría muy beneficiosa. El año 2012 el Comité Asesor de Vacunas e Inmunización (CAVEI) formuló la recomendación de: vacunar sólo a las niñas, en los colegios (para maximizar la cobertura), evaluar la factibilidad de vacunar mas de una cohorte de niñas entre 9 y 13 años y el aplicar 2 dosis de vacunas con esquema de 0 y 12 meses manteniendo una vigilancia epidemiológica a fin de decidir la necesidad futura de una tercera dosis a los 60 meses.

Desde Septiembre de este año el Ministerio de Salud de Chile, inició la vacunación de niñas de 4 básico (9 años) con un esquema de 0 y 12 meses con la vacuna cuadrivalente, estableciendo la necesidad de inmunizar no solo contra las patologías neoplásicas sino que también para cubrir la problemática de las verrugas genitales y alcanzado niveles de cobertura adecuados (sobre el $80 \%$ ). Esta política se enmarca dentro del programa nacional de inmunización e incluye no desatender el tamizaje para los grupos no cubiertos tal como establece la guía clínica nacional de cáncer de cuello uterino.

Sin embargo, la historia no termina y continúa escribiéndose. El futuro con seguridad nos deparará muchas sorpresas con probables cambios en los métodos de tamizaje, basados en técnicas moleculares de detección viral, la aparición de nuevas y mejores vacunas y quizás con un aumento de la población a vacunar, ya sea extendiendo la cobertura a otras edades o extendiéndola al sexo masculino. Independiente de ello, lo más relevante es que ya hoy contamos con una nueva herramienta para combatir una enfermedad que mata 2 mujeres diariamente en nuestro país y una cada dos minutos en el mundo.

Dr. Omar Nazzal Nazal. Ginecólogo Oncólogo. Profesor Asociado.

Director de Departamento de Obstetricia y Ginecología. Campus Centro, Facultad de Medicina, Universidad de Chile.

Dr. Mauricio Cuello Fredes.

Facultad de Medicina, Pontificia Universidad Católica de Chile. Ginecólogo Oncólogo. Profesor Asociado.

Jefe de Programa de Especialización en Obstetricia y Ginecología

\section{REFERENCIAS}

1. Brotherton JM, Fridman M, May CL, Chappell G, Saville AM, Gertig DM. Early effect of the HPV vaccination programme on cervical abnormalities in Victoria, Australia: an ecological study. Lancet 2011;377(9783):2085-92.

2. Chow EP, Read TR, Wigan R, Donovan B, Chen MY, Bradshaw CS, Fairley CK. Ongoing decline in genital warts among young heterosexuals 7 years after the Australian human papillomavirus (HPV) vaccination programme. Sex Transm Infect 2014. pii: sextrans-2014-051813. doi: $10.1136 /$ sextrans-2014-051813. 
3. Draper E, Bissett SL, Howell-Jones R, Edwards D, Munslow G, Soldan K, Beddows S. Neutralization of non-vaccine human papilloma virus pseudoviruses from the A7 and A9 species groups by bivalent HPV vaccine sera. Vaccine 2011;29(47):8585-90.

4. Dobson SR, McNeil S, Dionne M, Dawar M, Ogilvie G, Krajden M, Sauvageau C, Scheifele DW, Kollmann TR, Halperin SA, Langley JM, Bettinger JA, Singer J, Money D, Miller D, Naus M, Marra F, Young E. Immunogenicity of 2 doses of HPV vaccine in younger adolescents vs 3 doses in young women: a randomized clinical trial. JAMA 2013;309(17):1793-802.

5. Lehtinen M, Paavonen J, Wheeler CM, Jaisamrarn U, Garland SM, Castellsagué X, Skinner SR, Apter D, Naud P, Salmerón J, Chow SN, Kitchener H, Teixeira JC, Hedrick J, Limson G, Szarewski A, Romanowski B, Aoki FY, Schwarz TF, Poppe WA, De Carvalho NS, Germar MJ, Peters K, Mindel A, De Sutter P, Bosch FX, David MP, Descamps D, Struyf F, Dubin G; HPV PATRICIA Study Group. Overall efficacy of HPV-16/18 AS04-adjuvanted vaccine against grade 3 or greater cervical intraepithelial neoplasia: 4-year end-of-study analysis of the randomised, double-blind PATRICIA trial. Lancet Oncol 2012;13(1):89-99.

6. Muñoz N, Kjaer SK, Sigurdsson K, Iversen OE, Hernandez-Avila M, Wheeler CM, Perez G, Brown DR, Koutsky LA, Tay EH, Garcia PJ, Ault KA, Garland SM, Leodolter S, Olsson SE, Tang GW, Ferris DG, Paavonen J, Steben M, Bosch FX, Dillner J, Huh WK, Joura EA, Kurman RJ, Majewski S, Myers ER, Villa LL, Taddeo FJ, Roberts C, Tadesse A, Bryan JT, Lupinacci LC, Giacoletti KE, Sings HL, James MK, Hesley TM, Barr E, Haupt RM. Impact of human papillomavirus (HPV)-6/11/16/18 vaccine on all HPV-associated genital diseases in young women. $J$ Natl Cancer Inst 2010;102(5):325-39.

7. Neuzil KM, Canh do G, Thiem VD, Janmohamed A, Huong VM, Tang Y, Diep NT, Tsu V, LaMontagne DS.. Immunogenicity and reactogenicity of alternative schedules of HPV vaccine in Vietnam: a cluster randomized noninferiority trial. JAMA 2011;305(14):1424-31.

8. Paavonen J, Naud P, Salmerón J, Wheeler CM, Chow $\mathrm{SN}$, Apter D, Kitchener $\mathrm{H}$, Castellsague X, Teixeira JC, Skinner SR, Hedrick J, Jaisamrarn U, Limson G, Garland S, Szarewski A, Romanowski B, Aoki FY, Schwarz TF, Poppe WA, Bosch FX, Jenkins D, Hardt $\mathrm{K}$, Zahaf T, Descamps D, Struyf F, Lehtinen M, Dubin G; HPV PATRICIA Study Group. Efficacy of human papillomavirus (HPV)-16/18 AS04-adjuvanted vaccine against cervical infection and precancer caused by oncogenic HPV types (PATRICIA): final analysis of a double-blind, randomised study in young women. Lancet 2009;374(9686):301-14.

9. Stanley MA, Sudenga SL, Giuliano AR. Alternative dosage schedules with HPV virus-like particle vaccines. Expert Rev Vaccines 2014;13(8):1027-38.

10. WHO. Human papillomavirus vaccines: WHO position paper, October 2014. Weekly epidemiological record. 24 october 2014, 89th year 2014; 89:465-92. Disponible en: http://www.who.int/wer/2014/wer8943. pdf?ua $=1$.

11. FIGO Statement on HPV Vaccination Safety, August 2nd, 2013. Disponible en: http://www.figo.org/ sites/default/files/uploads/project-publications/Statement $\% 20$ on\%20Safety\%20of\%20HPV\%20vaccination\%20-\%20FINAL\%20-\%20AUGUST\%202013_0. pdf. 